\title{
Research Article \\ Extended Stokes' First Problem of an Oldroyd-B Fluid for Relatively Moving Half-Planes
}

\author{
Chi-Min Liu \\ Division of Mathematics, General Education Center, Chienkuo Technology University, \\ 500 Changhua City, Taiwan \\ Correspondence should be addressed to Chi-Min Liu, liu.chimin@gmail.com
}

Received 20 October 2010; Accepted 31 January 2011

Academic Editor: Horst Ecker

Copyright (C) 2011 Chi-Min Liu. This is an open access article distributed under the Creative Commons Attribution License, which permits unrestricted use, distribution, and reproduction in any medium, provided the original work is properly cited.

\begin{abstract}
An Oldroyd-B fluid suddenly disturbed by relatively moving half-planes is theoretically studied in this paper. This new problem, extended from the traditional Stokes' problem, recently attracts a great deal of attention due to its potential applications in engineering. Using integral transformations and dividing the original system into two subsystems, the exact solution is derived and shown in a series form. In addition to the general understanding of velocity developments, the effects of the relaxation time and the retardation time on the velocity profiles are examined in a series of figures. It is found that above two rheological parameters influence the induced flow in an opposite way. Other interesting characteristics are also elucidated from present results.
\end{abstract}

\section{Introduction}

The most classical problem in fluid mechanics might be a viscous flow driven by an impulsive or oscillatory plate below. This problem usually bears Stokes' name [1] due to his great contributions and profound influences on related studies. Today solutions of the Stokes' problems have been applied to many fields, which include the industry manufacturing, chemical engineering, geophysical flows, and even the heat conduction problems. For a Newtonian fluid, exact solutions to velocity profiles can be obtained using integral transforms (e.g., Panton [2] and Erdogan [3]) and the solution to wall stress in terms of Fresnel integrals was recently given by C. M. Liu and I. C. Liu [4].

For the rapidly growing applications either in academic studies or engineering fields, the studies on the Stokes' problems traditionally for a Newtonian fluid were extended to cases of non-Newtonian fluids. Since the rheological properties vary within a quite wide range, how to choose a suitable model to accurately describe fluid properties always becomes 
an important issue in related studies. To this end, the Maxwell model which is one of the simplest models for viscoelastic fluids was studied to understand basic fluid characteristics. Fetecau and Fetecau [5] solved the first Stokes' problem of a Maxwell fluid by applying the Fourier transform. Later, Jordan et al. [6] revisited the same problem by applying both Laplace and Fourier transform methods. In addition to Maxwell fluids, another common fluid, the second-grade fluid, was frequently investigated as well. Erdogan [7] and Tan and Masuoka [8] solved the first problem of a second-grade fluid. The second problem was later studied by Fetecau and Fetecau [9]. More recently, a series of papers concerning the first problem of Burger' fluids were also presented [10,11].

The Oldroyd-B fluid model [12], which includes the Newtonian fluid, the Maxwell fluid and the second-grade fluid as special cases, was also widely studied in the past decades. Solution to the Stokes' first problem of an Oldroyd-B fluid was given by Fetecau and Fetecau [13], and also by Tan and Masuoka [14] with the consideration of a porous boundary. Aksel et al. [15] later provided an analysis of the second problem and other unidirectional flows. Qi and $\mathrm{Xu}$ [16] considered a generalized Oldroyd-B model by applying the fractional calculus approach in the constitutive relationship. Their solution to the first problem was shown in a series form. Other specific flows were also well investigated by Rajagopal and Bhatnagar [17] and Hayat et al. [18].

In addition to studies contributing to various fluids based on various models, more boundary conditions were involved to meet the practical requirements. The most common modified boundary condition might be a porous plate which allows the mass injection and suction through the plate. The corresponding solutions have been obtained for different fluids $[8,14,19,20]$. Pressure gradient [9] and heated boundary were also studied [8]. For practical applications in predicting flows induced by either earthquakes or fracture of ice sheets, Zeng and Weinbaum [21] analyzed the extended Stokes' problems for a Newtonian fluid by imposing relatively moving half-planes as a boundary condition. They provided the exact solution for the first problem and the steady-state solution for the second problem which is accurate enough for long-term phenomena. For capturing the characteristics of flows at earlier stage, the transient solution to the second problem, however, is still lacking. To this end, C. M. Liu [22] derived the exact solution by using integral transforms and the concept of antisymmetry. His exact solution can calculate the flow not only at larger times, but also at smaller times. The finite-depth cases as well as the infinite-depth cases were investigated to calculate the velocity distributions.

In this paper, the flow of an Oldroyd-B fluid driven by relatively moving half-planes with constant speed is analyzed. This problem is named as the extended Stokes' first problem. The organization of present paper is as follows. The constitutive equations for the present problem are firstly derived in Section 2 . The detailed derivation and mathematical methods used are shown in Section 3. In addition to integral transforms, the main mathematical techniques include the division of the original fluid system into two subsystems and the expansion of the specific functions into series forms. Results are discussed in Section 4, and concluding remarks are made in Section 5.

\section{Constitutive Equations}

The flow field considered is depicted as Figure 1. An Oldroyd-B fluid rather than a Newtonian fluid [22] is considered to occupy all the space above the plate. The fluid and the plate are initially at rest everywhere. For $t>0$, the positive half-plate $(z>0)$ located at 


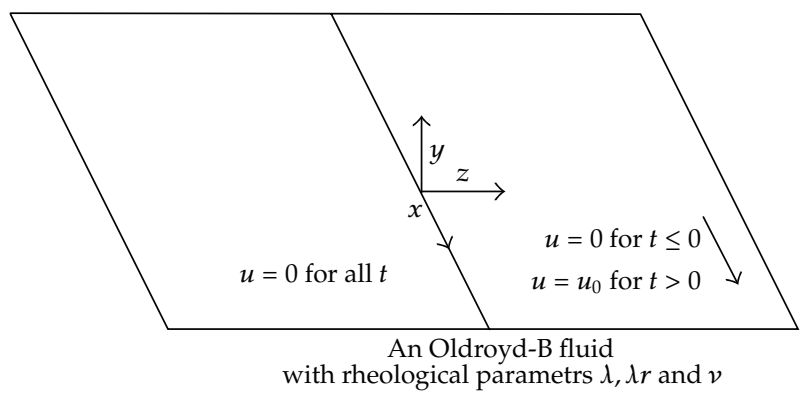

Figure 1: Diagram of an Oldroyd-B fluid driven by relatively moving half-planes.

$y=0$ is suddenly moving at a constant speed $u_{0}$ along the $x$ coordinate and the negative half-plate $(z<0)$ remains still. This is the so-called extended Stokes' first problem. Firstly, the constitutive equations for an incompressible Oldroyd-B fluid [23] are introduced

$$
\begin{aligned}
\mathbf{T} & =-p \mathbf{I}+\mathbf{S} \\
\mathbf{S}+\lambda\left(\dot{\mathbf{S}}-\mathbf{L S}-\mathbf{S L}^{\mathrm{T}}\right) & =\mu\left(\mathbf{A}+\lambda_{r}\left(\dot{\mathbf{A}}-\mathbf{L A}-\mathbf{A L}^{\mathrm{T}}\right)\right)
\end{aligned}
$$

where $\mathbf{T}$ denotes the Cauchy stress tensor, $\mathbf{S}$ the extra-stress tensor, $p$ the pressure, $\mathbf{I}$ the isotropic unit tensor, $\mathbf{L}=\nabla \mathbf{V}$ where $\mathbf{V}$ is the velocity and $\nabla$ is the gradient operator, and A is defined to be $\mathbf{A}=\mathbf{L}+\mathbf{L}^{\mathrm{T}}$. The fluid constants $\mu, \lambda$, and $\lambda_{r}$ are the dynamic viscosity, the relaxation time, and the retardation times, respectively. The superposed dot represents the material time derivative. This model includes several fluid models as special cases. They are the Maxwell fluid $\left(\lambda_{r}=0\right)$ and the Newtonian fluid $\left(\lambda=\lambda_{r}=0\right)$. Since the fluid is assumed to be incompressible, it leads to

$$
\operatorname{div} \mathbf{V}=0
$$

As the flow only moves along the $x$ direction, we assume

$$
\mathbf{V}=u(y, z, t) \mathbf{i}
$$

where $\mathbf{i}$ represents a unit vector along the $x$ direction. Substituting (2.4) into the linear momentum equation and neglecting the body forces, it reads

$$
\frac{\partial p}{\partial x}+\frac{\partial S_{x x}}{\partial x}+\frac{\partial S_{x y}}{\partial y}+\frac{\partial S_{x z}}{\partial z}=\rho \frac{\partial u}{\partial t}
$$

where $\rho$ denotes the density of the fluid. For the present flow, it is noted that all physical variables are independent of $x$ implying the first two terms of (2.5) are zero. From (2.1), 
relations between $S_{x x}, S_{y y}, S_{z z}, S_{x y}\left(=S_{y x}\right), S_{y z}\left(=S_{z y}\right)$, and $S_{x z}\left(=S_{z x}\right)$ are

$$
\begin{gathered}
S_{x x}+\lambda\left(\frac{\partial S_{x x}}{\partial t}-2 S_{x y} \frac{\partial u}{\partial y}-2 S_{x z} \frac{\partial u}{\partial z}\right)=-2 \mu \lambda_{r}\left[\left(\frac{\partial u}{\partial y}\right)^{2}+\left(\frac{\partial u}{\partial z}\right)^{2}\right] \\
S_{x y}+\lambda\left(\frac{\partial S_{x y}}{\partial t}-S_{y y} \frac{\partial u}{\partial y}-S_{y z} \frac{\partial u}{\partial z}\right)=\mu \frac{\partial u}{\partial y}+\mu \lambda_{r} \frac{\partial^{2} u}{\partial y \partial t} \\
S_{x z}+\lambda\left(\frac{\partial S_{x z}}{\partial t}-S_{y z} \frac{\partial u}{\partial y}-S_{z z} \frac{\partial u}{\partial z}\right)=\mu \frac{\partial u}{\partial z}+\mu \lambda_{r} \frac{\partial^{2} u}{\partial z \partial t} \\
S_{y y}+\lambda \frac{\partial S_{y y}}{\partial t}=0 \\
S_{y z}+\lambda \frac{\partial S_{y z}}{\partial t}=0 \\
S_{z z}+\lambda \frac{\partial S_{z z}}{\partial t}=0
\end{gathered}
$$

By considering the initial condition $\mathbf{S}(t=0)=0$, solutions to (2.8) to (2.10) can be solved

$$
S_{y y}=S_{y z}=S_{z z}=0
$$

By using (2.12), (2.7) and (2.8) can be simplified to be

$$
\begin{aligned}
& S_{x y}+\lambda \frac{\partial S_{x y}}{\partial t}=\mu\left(1+\lambda_{r} \frac{\partial}{\partial t}\right) \frac{\partial u}{\partial y} \\
& S_{x z}+\lambda \frac{\partial S_{x z}}{\partial t}=\mu\left(1+\lambda_{r} \frac{\partial}{\partial t}\right) \frac{\partial u}{\partial z} .
\end{aligned}
$$

Now we combine (2.5), (2.6), and (2.13) together to obtain the final constitutive equation

$$
\left(1+\lambda \frac{\partial}{\partial t}\right) \frac{\partial u}{\partial t}=v\left(1+\lambda_{r} \frac{\partial}{\partial t}\right)\left(\frac{\partial^{2} u}{\partial y^{2}}+\frac{\partial^{2} u}{\partial z^{2}}\right)
$$

where $v=\mu / \rho$ denotes the kinematic viscosity. In comparison with the traditional Stokes' problems of an Oldroyd-B fluid, the additional terms of $\partial^{2} u / \partial z^{2}$ appear. The detailed transform methods as well as other mathematical techniques for solving (2.14) and the associated conditions will be demonstrated in the following section. 


\section{Transform Methods and Solutions}

For the flow system shown in Figure 1, the governing equation, the associated boundary conditions, and initial conditions are shown below

$$
\begin{gathered}
u(y=0, z>0, t)=u_{0}, \\
u(y=0, z<0, t)=0, \\
u(y \longrightarrow \infty, z, t)=0, \\
u(y, z \longrightarrow \pm \infty, t) \text { is finite, } \\
u(y, z, t=0)=\frac{\partial u}{\partial t}(y, z, t=0)=0,
\end{gathered}
$$

where $u_{0}$ is the constant plate speed. To solve this flow system, the original problem is decomposed into two subproblems

$$
u=u_{1}+u_{2}
$$

where $u_{1}$ and $u_{2}$, respectively, satisfy

$$
\begin{gathered}
\left(1+\lambda \frac{\partial}{\partial t}\right) \frac{\partial u_{1}}{\partial t}=v\left(1+\lambda_{r} \frac{\partial}{\partial t}\right) \frac{\partial^{2} u_{1}}{\partial y^{2}} \\
\left(1+\lambda \frac{\partial}{\partial t}\right) \frac{\partial u_{2}}{\partial t}=v\left(1+\lambda_{r} \frac{\partial}{\partial t}\right)\left(\frac{\partial^{2} u_{2}}{\partial y^{2}}+\frac{\partial^{2} u_{2}}{\partial z^{2}}\right)
\end{gathered}
$$

and are respectively constrained by

$$
\begin{gathered}
u_{1}(y=0, t)=\frac{u_{0}}{2}, \\
u_{1}(y \longrightarrow \infty, t)=0, \\
u_{1}(y, t=0)=\frac{\partial u_{1}}{\partial t}(y, t=0)=0,
\end{gathered}
$$

and

$$
\begin{gathered}
u_{2}(y=0, z>0, t)=\frac{u_{0}}{2}, \\
u_{2}(y=0, z<0, t)=-\frac{u_{0}}{2}, \\
u_{2}(y \longrightarrow \infty, z, t)=0, \\
u_{2}(y, z \longrightarrow \pm \infty, t) \text { is finite, } \\
u_{2}(y, z, t=0)=\frac{\partial u_{2}}{\partial t}(y, t=0)=0 .
\end{gathered}
$$


The solution to $u_{1}$, which is for the traditional Stokes' first problem, is first introduced. Applying the Laplace transform

$$
\widehat{u}(y, s)=\int_{0}^{\infty} e^{-s t} u(y, t) \mathrm{dt},
$$

to (3.3), (3.5) and (3.6) with the help of (3.7) results in

$$
\begin{gathered}
\frac{\partial^{2} \widehat{u}_{1}}{\partial y^{2}}=\frac{s(1+\lambda s)}{v\left(1+\lambda_{r} s\right)} \widehat{u}_{1}, \\
\widehat{u}_{1}(y=0, s)=\frac{u_{0}}{2 s}, \\
\widehat{u}_{1}(y \longrightarrow \infty, s)=0 .
\end{gathered}
$$

Thus, the solution to $\widehat{u}_{1}$ is

$$
\widehat{u}_{1}(y, s)=\frac{u_{0}}{2 s} \exp \left[-y \sqrt{\frac{s(1+\lambda s)}{v\left(1+\lambda_{r} s\right)}}\right] .
$$

In order to obtain an analytical solution and avoid the tedious calculations of residues and contour integrals, (3.15) is rewritten in a series form

$$
\begin{array}{r}
\widehat{u}_{1}(y, s)=\frac{u_{0}}{2}\left[\frac{1}{s}+\sum_{k=1}^{\infty} \sum_{m=0}^{\infty} \sum_{n=0}^{\infty} \frac{\left(-y \sqrt{\lambda / v \lambda_{r}}\right)^{k}(-\lambda)^{-m}\left(-\lambda_{r}\right)^{-n}}{k ! m ! n !}\right. \\
\left.\cdot \frac{\Gamma((k / 2)+n) \Gamma(-(k / 2)+m)}{\Gamma(k / 2) \Gamma(-k / 2)} \cdot s^{(k / 2)-m-n-1}\right],
\end{array}
$$

where ! denotes the factorial and $\Gamma$ the Gamma function. By applying the inverse Laplace transform to (3.16), it yields

$$
\begin{aligned}
& u_{1}(y, t)=\frac{u_{0}}{2}\left[1+\sum_{k=1}^{\infty} \sum_{m=0}^{\infty} \sum_{n=0}^{\infty} \frac{\left(-y \sqrt{\lambda / v \lambda_{r}}\right)^{k}(-\lambda)^{-m}\left(-\lambda_{r}\right)^{-n}}{k ! m ! n !}\right. \\
&\left.\frac{\Gamma((k / 2)+n) \Gamma(-(k / 2)+m)}{\Gamma(k / 2) \Gamma(-k / 2)} \cdot \frac{t^{-(k / 2)+m+n}}{\Gamma(-(k / 2)+m+n+1)}\right] .
\end{aligned}
$$


To rewrite (3.17) in a dimensionless form, substituting the following dimensionless parameters

$$
u^{*}=\frac{u}{u_{0}}, \quad y^{*}=\frac{u_{0} y}{v}, \quad t^{*}=\frac{u_{0}^{2} t}{v}, \quad \lambda^{*}=\frac{u_{0}^{2} \lambda}{v}, \quad \lambda_{r}^{*}=\frac{u_{0}^{2} \lambda_{r}}{v}
$$

into (3.17) results in

$$
\begin{aligned}
u_{1}(y, t)=\frac{1}{2}\left[1+\sum_{k=1}^{\infty} \sum_{m=0}^{\infty} \sum_{n=0}^{\infty} \frac{\left(-y \sqrt{\lambda / \lambda_{r}}\right)^{k}(-\lambda)^{-m}\left(-\lambda_{r}\right)^{-n}}{k ! m ! n !}\right. \\
\left.\cdot \frac{\Gamma((k / 2)+n) \Gamma(-(k / 2)+m)}{\Gamma(k / 2) \Gamma(-k / 2)} \cdot \frac{t^{-(k / 2)+m+n}}{\Gamma(-(k / 2)+m+n+1)}\right],
\end{aligned}
$$

where all asterisks are dropped for convenience. As for the second subsystem of $u_{2}$, since the positive- and negative- $z$ plates move with the same speed but in the opposite directions, the induced flow which is antisymmetrical with respect to $z=0$ satisfies

$$
u_{2}(y,+z, t)=-u_{2}(y,-z, t)
$$

which requires

$$
u_{2}(y, z=0, t)=0
$$

Above results imply that the velocity $u_{2}$ in the whole domain can be calculated by only solving the flow in the positive- $z$ domain. For flows in the positive- $z$ domain, the boundary conditions of (3.9) and (3.11) can be replaced by

$$
\begin{gathered}
u_{2}(y, z=0, t>0)=0, \\
u_{2}(y, z \longrightarrow \infty, t) \text { is finite. }
\end{gathered}
$$

Since the velocity $u_{2}$ varies with the time $t$ and the space coordinates $y$ and $z$, in addition to the Laplace transform, it is noted that an additional integral transform in space coordinate is needed to obtain the solvable boundary-value problem. Firstly, applying the Laplace transform to (3.4), (3.8), (3.10), and (3.22) with the help of (3.12) yields

$$
\begin{gathered}
\frac{\partial^{2} \widehat{u}_{2}}{\partial y^{2}}+\frac{\partial^{2} \widehat{u}_{2}}{\partial z^{2}}=\frac{s(1+\lambda s)}{v\left(1+\lambda_{r} s\right)} \widehat{u}_{2} \\
\widehat{u}_{2}(y=0, z, s)=\frac{u_{0}}{2 s} \\
\widehat{u}_{2}(y \longrightarrow \infty, z, s)=0
\end{gathered}
$$




$$
\begin{gathered}
\widehat{u}_{2}(y, z=0, s)=0, \\
\widehat{u}_{2}(y, z \longrightarrow \infty, s) \text { is finite. }
\end{gathered}
$$

Now one further applies the Fourier sine transform

$$
\tilde{u}(\omega, z, s)=\int_{0}^{\infty} \widehat{u}_{2}(y, z, s) \sin (\omega y) \mathrm{d} y,
$$

to (3.23); it yields

$$
\begin{gathered}
\frac{\partial^{2} \tilde{u}_{2}}{\partial z^{2}}-\left(\alpha^{2}+\omega^{2}\right) \tilde{u}_{2}=-\frac{u_{0} \omega}{2 s} \\
\tilde{u}_{2}(\omega, z=0, s)=0 \\
\tilde{u}_{2}(\omega, z \longrightarrow \infty, s) \text { is finite }
\end{gathered}
$$

where

$$
\alpha^{2}=\frac{s(1+\lambda s)}{v\left(1+\lambda_{r} s\right)}
$$

By solving (3.25), $\tilde{u}_{2}$ is readily obtained

$$
\tilde{u}_{2}(\omega, z, s)=\frac{u_{0} \omega}{2 s\left(\omega^{2}+\alpha^{2}\right)}\left(1-e^{-\sqrt{\omega^{2}+\alpha^{2}} \cdot z}\right) .
$$

Above equation can be further rewritten in a series form

$$
\tilde{u}_{2}=-\frac{u_{0}}{2 s} \sum_{k=1}^{\infty} \frac{(-z)^{k} \omega\left(\omega^{2}+\alpha^{2}\right)^{(k / 2)-1}}{k !} .
$$

After applying the inverse Fourier sine transforms to (3.28), the result is

$$
\widehat{u}_{2}=-\frac{u_{0}}{2 s \sqrt{\pi}} \sum_{k=1}^{\infty}\left(-\frac{z}{y}\right)^{k}(2 \alpha y)^{(1 / 2)(1+k)} \frac{\mathrm{K}_{0.5(1+k)}(\alpha y)}{k ! \Gamma(1-k / 2)},
$$

where $\mathrm{K}$ is the modified Bessel function of second kind. After applying the calculation shown in the appendix, the dimensionless form for $\widehat{u}_{2}$ can be obtained by using the dimensionless parameters defined in (3.18) as well as a new parameter $z^{*}=u_{0} z / v$

$$
\begin{aligned}
\widehat{u}_{2}= & -\frac{1}{2 \sqrt{\pi}} \sum_{n=1}^{\infty} \frac{(-z / y)^{2 n-1} 2^{n}}{(2 n-1) ! \Gamma(3 / 2-n)} \\
& \cdot\left\{\sum_{m=0}^{n-1} \sum_{p=0}^{\infty} \sum_{q=0}^{\infty} \frac{\left(-y^{2}\right)^{m}(n-m-1) !}{2^{2 m-n+1} m !}\left(\frac{\lambda}{\lambda_{r}}\right)^{m} \frac{(-\lambda)^{p}}{p !} \frac{\left(-\lambda_{r}\right)^{q}}{q !} \frac{\Gamma(-m+p)}{\Gamma(-m)} \frac{\Gamma(m+q)}{\Gamma(m)} s^{m-p-q-1}\right.
\end{aligned}
$$




$$
\begin{aligned}
& +(-1)^{n+1} \sum_{m=0}^{\infty} \sum_{p=0}^{\infty} \sum_{q=0}^{\infty} \frac{y^{2 m+2 n}}{2^{2 m+n} m !(m+n) !}\left(\frac{\lambda}{\lambda_{r}}\right)^{n+m} \frac{(-\lambda)^{p}}{p !} \frac{\left(-\lambda_{r}\right)^{q}}{q !} \frac{\Gamma(-m-n+p)}{\Gamma(-m-n)} \\
& \left.\times \frac{\Gamma(m+n+q)}{\Gamma(m+n)} \cdot s^{m+n-p-q-1} \cdot\left[\frac{1}{2} \ln \frac{s(1+\lambda s)}{\left(1+\lambda_{r} s\right)}+\ln \frac{y}{2}-\frac{1}{2} \varphi(m+1)-\frac{1}{2} \varphi(m+n+1)\right]\right\} \\
& -\frac{1}{2} \sum_{n=1}^{\infty} \sum_{m=0}^{n} \sum_{k=0}^{\infty} \sum_{p=0}^{\infty} \sum_{q=0}^{\infty} \frac{(-z)^{2 n} y^{-m-n+k} 2^{n-m}(m+n) !}{(2 n) ! m !(n-m) ! \Gamma(1-n)} \frac{(-1)^{k}}{k !}\left(\frac{\lambda}{\lambda_{r}}\right)^{(1 / 2)(n+k-m)} \frac{(-\lambda)^{p}}{p !} \frac{\left(-\lambda_{r}\right)^{q}}{q !} \\
& \cdot \frac{\Gamma(-(1 / 2)(n+k-m)+p)}{\Gamma(-(1 / 2)(n+k-m))} \frac{\Gamma((1 / 2)(n+k-m)+q)}{\Gamma((1 / 2)(n+k-m))} \cdot s^{0.5(n+k-m)-p-q-1},
\end{aligned}
$$

where all asterisks for dimensionless variables are dropped. The final step of the derivation is to apply the Laplace inverse transform to (3.30). To this end, terms containing the transform variable $s$ in (3.30) are classified into two kinds. For terms only involving powers of $s$, it requires the following rules of inverse Laplace transform to calculate the inversion

$$
\begin{gathered}
L^{-1}\left(s^{n}\right)= \begin{cases}\frac{t^{\mathrm{Abs}(n)-1}}{(\operatorname{Abs}(n)-1) !} & \text { for } n=-1,-2,-3, \ldots \\
\frac{\mathrm{d}^{n}}{\mathrm{~d} t^{n}} \delta(t) & \text { for } n=0,1,2, \ldots,\end{cases} \\
L^{-1}\left(s^{n-(1 / 2)}\right)= \begin{cases}\frac{4^{\mathrm{Abs}(n)}}{\sqrt{\pi}} \frac{(\operatorname{Abs}(n)) !}{(2 \operatorname{Abs}(n)) !} t^{-(1 / 2)+\operatorname{Abs}(n)} & \text { for } n=-1,-2,-3, \ldots \\
\frac{4^{n}}{\sqrt{\pi}} \frac{n !}{(2 n) !} \int_{0}^{\infty}\left(t^{\prime}\right)^{n-(1 / 2)} \mathrm{J}_{0}\left(2 \sqrt{t^{\prime} t}\right) \mathrm{d} t^{\prime} & \text { for } n=0,1,2, \ldots,\end{cases}
\end{gathered}
$$

where $\delta(t)$ represents the Dirac delta function, $\mathrm{J}(t)$ indicates the Bessel function of the first kind and $\operatorname{Abs}(n)$ the absolute value. As for terms containing the product of powers and the logarithm of $s$, the inversion will be calculated in a convolution form with the help of

$$
L^{-1}[\ln (1+a s)]=\frac{-\exp (-t / a)}{t}
$$

Finally, by combing the solutions of $u_{1}$ and $u_{2}$, the velocity distribution either in the positiveor the negative- $z$ domain can be analytically calculated.

In this section, the total solution consisting of $u_{1}$ and $u_{2}$ will be calculated and plotted in dimensionless forms. The focus of the analysis is how the rheological parameters influence 


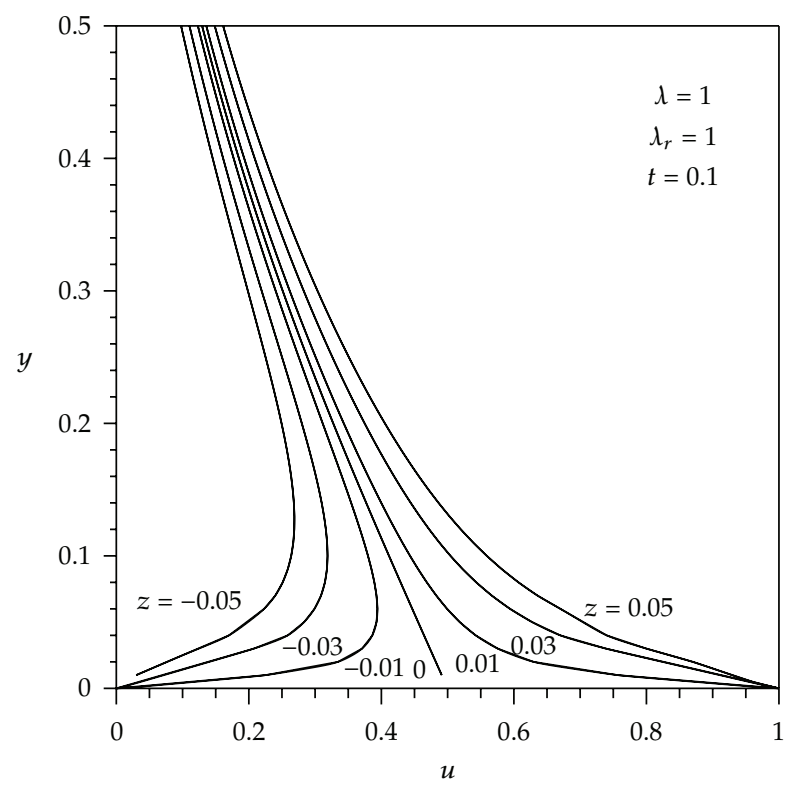

Figure 2: Velocity profiles at various $z$ sections. The rheological parameters are $\lambda=\lambda_{r}=1$.

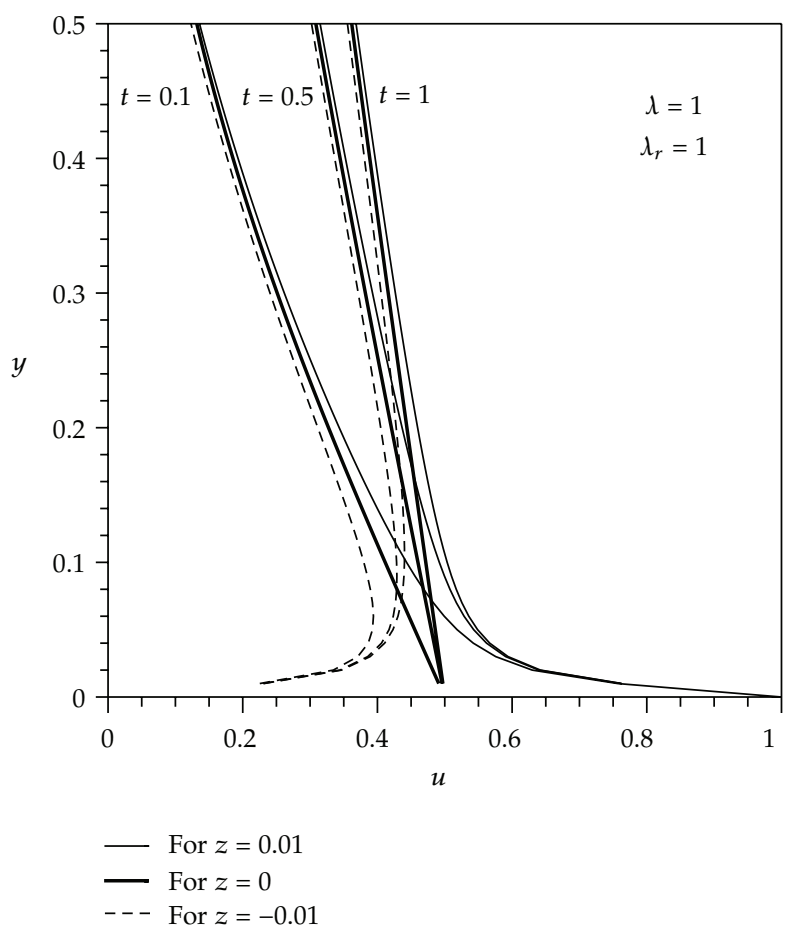

Figure 3: Velocity profiles for $z=0.01$ (solid), $z=0$ (broad-solid), and $z=-0.01$ (dashed) at $t=0.1,0.5$, and 1.0. The rheological parameters are $\lambda=\lambda_{r}=1$. 


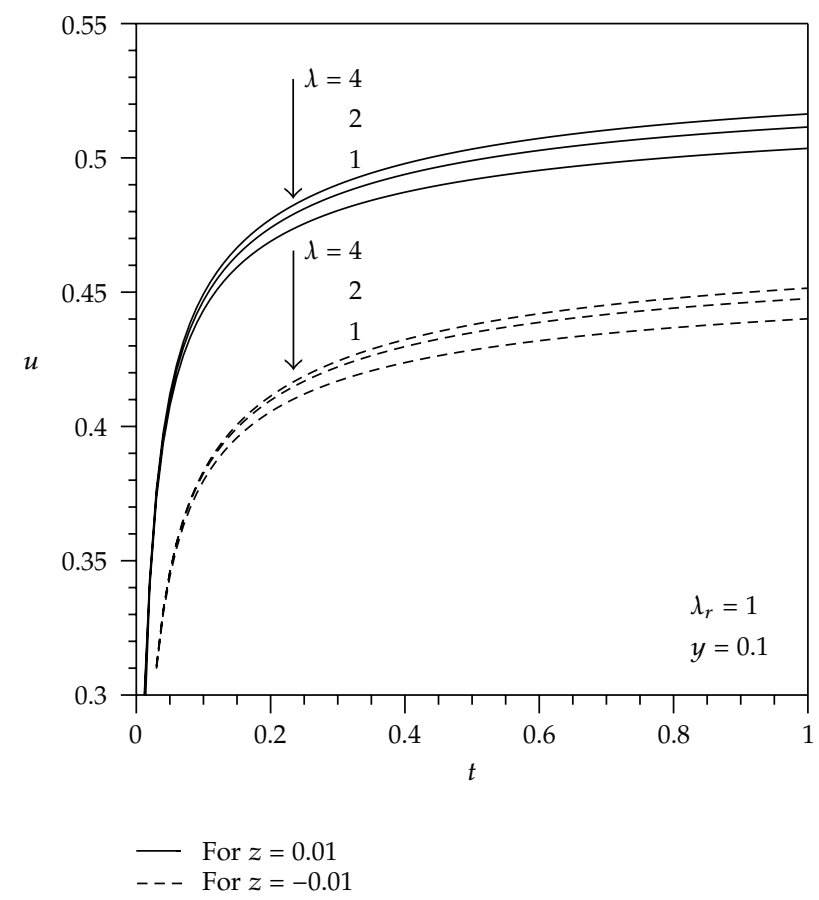

Figure 4: Velocity developments at $z=0.01$ (solid) and $z=-0.01$ (dashed) for the cases of $\lambda=1,2$, and 4 at the level $y=0.1$. The fixed retardation time is $\lambda_{r}=1$.

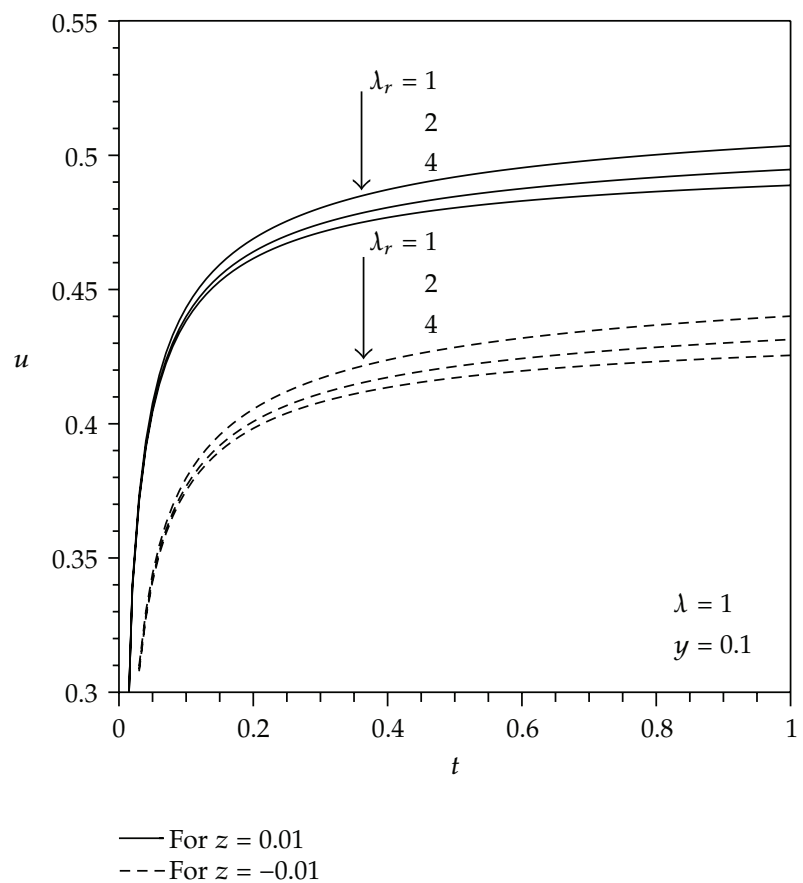

Figure 5: Velocity developments at $z=0.01$ (solid) and $z=-0.01$ (dashed) for the cases of $\lambda_{r}=1,2$, and 4 at the level $y=0.1$. The fixed relaxation time is $\lambda=1$. 
the velocity profiles at various $z$ sections. Firstly, the velocity profiles at various $z$ sections for the conditions $\lambda=1, \lambda_{r}=1$, and $t=0.1$ are plotted in Figure 2. This figure gives a general understanding of flows driven by relatively moving half-planes. It indicates that for the positive $z$-plane, the influence of the still half-plate will decay as $z$ grows. Similarly, at the far end of the negative $z$-plane, the effect of the moving plane becomes much weaker. It is remarked that velocities at $z=0$ are calculated by the solution of the traditional Stokes' problem, and the singularity exists along the line $y=z=0$. To elucidate the velocity developments more clearly, the velocity profiles at $z=0.01,0$, and -0.01 at different times $\left(t=0.1,0.5\right.$ and 1.0) for the conditions $\lambda=1$ and $\lambda_{r}=1$ are plotted in Figure 3. Solid, broadsolid and dashed curves are drawn for the velocity distributions at $z=0.01,0$, and -0.01 , respectively. At the early stage, flows above the moving plane $(z>0)$ are quite different from those above the still plane $(z<0)$ as it has not enough time to fully deliver the effects of the moving plane and the still plane to flows above each other. It is also found that velocity profiles either at $z=0.01$ or at $z=-0.01$ will move with that at $z=0$ as time goes by.

\section{Results and Discussion}

In addition to a general understanding given by Figures 2 and 3, the effects of two important rheological parameters for an Oldroyd-B fluid, the relaxation time and the retardation time, on the velocity developments are investigated by plotting the velocity diagrams at $y=0.1$ level. Figure 4 shows the velocity developments for different relaxation times $(\lambda=1,2$, and 4) with a fixed retardation time $\lambda_{r}=1$. Solid and dashed curves denote the results at $z=0.01$ and $z=-0.01$, respectively. It is clearly seen that a larger value of $\lambda$ will result in a faster development of the velocity profile. Moreover, flows above the negative$z$ plane are much weaker than those above the positive- $z$ plane which can be also seen in Figure 2. On the other hand, the effects of changing the retardation time $\left(\lambda_{r}=1,2\right.$ and 4 ) on the velocity field are depicted in Figure 5. It is of interest that, in comparison with the relaxation time, the retardation time plays an opposite role in the flow development. Namely, a larger value of $\lambda_{r}$ will yield a slower growth of velocity profiles at both sides of $z$ half-planes.

\section{Concluding Remarks}

The extended Stokes' first problem of an Oldroyd-B fluid driven by relatively moving halfplanes is theoretically studied in this paper. Using integral transformations and dividing the original problem into two subsystems, the total solution is derived and shown in a series form. Velocity profiles and their developments influenced by the relaxation time and the retardation time are examined. From the present results, the following conclusions are summarized: (1) the velocity profiles above both half-planes will move with that at the central plane $(z=0),(2)$ a larger value of the relaxation time will result in a faster development of the velocity profile, and (3) the effects of the magnitude of the retardation time on the velocity field are opposite to those of the relaxation time. 


\section{Appendix}

In this appendix, the derivation of displaying (3.29) in a series form is presented. The key technique to acquire the inversion of (3.29) is to expand the Bessel function in a series form. First the corresponding identities of Bessel functions are introduced below [24]

$$
\begin{aligned}
\mathrm{K}_{n}(z)= & \frac{1}{2} \sum_{k=0}^{n-1} \frac{(-1)^{k}(z / 2)^{2 k-n}(n-k-1) !}{k !}+(-1)^{n+1} \sum_{k=0}^{\infty} \frac{(z / 2)^{n+2 k}}{k !(n+k) !} \\
& \times\left[\ln \left(\frac{z}{2}\right)-\frac{1}{2} \varphi(k+1)-\frac{1}{2} \varphi(n+k+1)\right] \quad \text { for } n=1,2,3, \ldots, \\
\mathrm{K}_{n}(z)= & \sqrt{\frac{\pi}{2 z}} \cdot e^{-z} \sum_{k=0}^{n-(1 / 2)} \frac{(2 z)^{-k}(n+k-1 / 2) !}{k !(n-k-1 / 2) !} \text { for } n=\frac{1}{2}, \frac{3}{2}, \frac{5}{2}, \ldots,
\end{aligned}
$$

where $\varphi$ is the digamma function defined as

$$
\begin{gathered}
\varphi(1)=-\gamma, \\
\varphi(n)=-\gamma+\sum_{k=1}^{n-1} k^{-1}, \quad n \geq 2, \\
\varphi\left(\frac{1}{2}\right)=-\gamma-2 \ln 2, \\
\varphi\left(n+\frac{1}{2}\right)=-\gamma-2 \ln 2+2\left(1+\frac{1}{3}+\cdots+\frac{1}{2 n-1}\right), \quad n \geq 2,
\end{gathered}
$$

where $n$ is integer and $\gamma$ is the Euler constant $(\gamma=0.5772 \ldots)$. Since there exist two kinds of expansion depending on the index $n$, Bessel functions in (3.29) will be classified and expanded in two different types. With the help of (A.1), terms of odd $k$ in (3.29) are collected and rewritten as

$$
\begin{aligned}
\widehat{u}_{2, \text { odd }}= & -\frac{u_{0}}{2 s \sqrt{\pi}} \sum_{k=1,3,5 \ldots}^{\infty}\left(-\frac{z}{y}\right)^{k} \frac{(2 \alpha y)^{(1 / 2)(1+k)}}{k ! \Gamma(1-k / 2)} \\
& \cdot\left\{\sum_{m=0}^{(1 / 2)(k-1)} \frac{(-1)^{m}}{2} \frac{[(1 / 2)(k-1)-m] !}{m !(\alpha y / 2)^{(1 / 2)(k+1)-2 m}}+(-1)^{(1 / 2)(k+3)}\right. \\
& \left.\times \sum_{m=0}^{\infty} \frac{(\alpha y / 2)^{(1 / 2)(k+1)+2 m}}{m ![(1 / 2)(k+1)+m] !}\left[\ln \left(\frac{\alpha y}{2}\right)-\frac{1}{2} \varphi(m+1)-\frac{1}{2} \varphi\left[\frac{1}{2}(k+3)+m\right]\right]\right\}
\end{aligned}
$$


and can be also shown as

$$
\begin{aligned}
\widehat{u}_{2, \text { odd }}= & -\frac{u_{0}}{s \sqrt{2 \pi}} \sum_{n=2,4,6, \ldots}^{\infty} \frac{(-\sqrt{2} z / y)^{n-1}}{(n-1) ! \Gamma((3-n) / 2)} \\
& \cdot\left\{\sum_{m=0}^{(1 / 2)(n-2)}\left(-\frac{y^{2}}{4}\right)^{m} \frac{[(1 / 2)(n-2)-m] !}{2^{(1-(n / 2))} m !}\left(\alpha^{2}\right)^{m}-(-2)^{n / 2}\right. \\
& \left.\times \sum_{m=0}^{\infty} \frac{(y / 2)^{n+2 m}\left(\alpha^{2}\right)^{(n / 2)+m}}{m !((1 / 2) n+m) !} \cdot\left[\frac{1}{2} \ln \left(\alpha^{2}\right)+\ln \frac{y}{2}-\frac{1}{2} \varphi(m+1)-\frac{1}{2} \varphi\left(\frac{1}{2} n+m+1\right)\right]\right\} .
\end{aligned}
$$

To perform the inverse transform from s-domain to $t$-domain, terms of $\alpha^{2}$ which contain the variable $s$ have to be expanded in a series form as well

$$
\left(\alpha^{2}\right)^{m}=\left(\frac{\lambda}{v \lambda_{r}}\right)^{m} \sum_{p=0}^{\infty} \sum_{q=0}^{\infty} \frac{(-\lambda)^{-p}}{p !} \frac{\left(-\lambda_{r}\right)^{-q}}{q !} \frac{\Gamma(m+q)}{\Gamma(m)} \frac{\Gamma(-m+p)}{\Gamma(-m)} \cdot s^{m-p-q},
$$

where $\Gamma$ denotes the Gamma function. Applying (A.6) into (A.5) yields

$$
\widehat{u}_{2, \text { odd }}=-\frac{u_{0}}{\sqrt{2 \pi}} \sum_{n=2,4,6, \ldots}^{\infty} \frac{(-\sqrt{2} z / y)^{n-1}}{(n-1) ! \Gamma((3-n) / 2)} \cdot\left(C_{1}+C_{2}+C_{3}\right),
$$

where

$$
\begin{gathered}
C_{1}=\sum_{m=0}^{(n / 2)-1} \sum_{p=0}^{\infty} \sum_{q=0}^{\infty}\left\{\left(-\frac{y^{2}}{4}\right)^{m} \frac{[(1 / 2)(n-2)-m] !}{2^{(1-(n / 2))} m !}\left(\frac{\lambda}{v \lambda_{r}}\right)^{m} \frac{(-\lambda)^{-p}}{p !} \frac{\left(-\lambda_{r}\right)^{-q}}{q !} \frac{\Gamma(m+q)}{\Gamma(m)}\right. \\
\left.\quad \frac{\Gamma(-m+p)}{\Gamma(-m)} \cdot s^{m-p-q-1}\right\}, \\
C_{2}=-(-2)^{n / 2} \sum_{m=0}^{\infty} \sum_{p=0}^{\infty} \sum_{q=0}^{\infty}\left\{\frac{(y / 2)^{n+2 m}\left(\lambda / v \lambda_{r}\right)^{(n / 2)+m}}{m !((1 / 2) n+m) !}\left[\ln \frac{y \sqrt{v}}{2}-\frac{1}{2} \varphi(m+1)-\frac{1}{2} \varphi\left(\frac{1}{2} n+m+1\right)\right]\right. \\
\left.\quad \cdot \frac{(-\lambda)^{-p}}{p !} \frac{\left(-\lambda_{r}\right)^{-q}}{q !} \frac{\Gamma((n / 2)+m+q)}{\Gamma((n / 2)+m)} \frac{\Gamma(-(n / 2)-m+p)}{\Gamma(-(n / 2)-m)} s^{(n / 2)+m-p-q-1}\right\}, \\
C_{3}=-\frac{(-2)^{n / 2}}{2} \sum_{m=0}^{\infty} \sum_{p=0}^{\infty} \sum_{q=0}^{\infty}\left\{\frac{(y / 2)^{n+2 m}\left(\lambda / v \lambda_{r}\right)}{m !((1 / 2) n+m) !} \frac{(-\lambda)^{-p}}{p !} \frac{\left(-\lambda_{r}\right)^{-q}}{q !} \frac{\Gamma((n / 2)+m+q)}{\Gamma((n / 2)+m)}\right. \\
\left.\quad \frac{\Gamma(-(n / 2)-m+p)}{\Gamma(-(n / 2)-m)} \ln \frac{s(1+\lambda s)}{1+\lambda_{r} s} s^{(n / 2)+m-p-q-1}\right\},
\end{gathered}
$$


where $C_{1}$ and $C_{2}$ only contain the powers of $s$ and $C_{3}$ has the products of logarithm and powers of $s$. Now the terms of even $k$ in (3.29) are expressed as

$$
\widehat{u}_{2, \text { even }}=-\frac{u_{0}}{2 s \sqrt{\pi}} \sum_{n=1}^{\infty}\left(-\frac{z}{y}\right)^{2 n} \frac{(2 \alpha y)^{n+(1 / 2)}}{(2 n) !} \frac{K_{n+(1 / 2)}(\alpha y)}{\Gamma(1-n)},
$$

and can be further rewritten as

$$
\widehat{u}_{2, \text { even }}=-\frac{u_{0}}{2 s} \sum_{n=1}^{\infty} \sum_{m=0}^{n} \frac{2^{n-m}(-z)^{2 n} y^{-n-m}}{\Gamma(1-n)} \frac{(n+m) !}{(2 n) ! m !(n-m) !} \alpha^{n-m} \exp (-\alpha y)
$$

Applying (A.6) into (A.10) leads to

$$
\begin{aligned}
\widehat{u}_{2, \text { even }}= & -\frac{u_{0}}{2} \sum_{n=1}^{\infty} \sum_{m=0}^{n} \sum_{k=0}^{\infty} \sum_{p=0}^{\infty} \sum_{q=0}^{\infty} \frac{(-1)^{k} 2^{n-m}(-z)^{2 n} y^{-n-m+k}}{\Gamma(1-n)} \frac{(n+m) !}{k !(2 n) ! m !(n-m) !}\left(\frac{\lambda}{v \lambda_{r}}\right)^{(1 / 2)(n+k-m)} \\
& \cdot \frac{(-\lambda)^{-p}}{p !} \frac{\left(-\lambda_{r}\right)^{-q}}{q !} \frac{\Gamma[(1 / 2)(n+k-m)+q]}{\Gamma[(1 / 2)(n+k-m)]} \frac{\Gamma(-(1 / 2)(n+k-m)+p)}{\Gamma[-(1 / 2)(n+k-m)]} s^{(1 / 2)(n+k-m)-p-q-1,},
\end{aligned}
$$

where only the powers of $s$ exist for the inverse calculation. Finally, (3.29) can be rewritten by using the summation of (A.6) and (A.11) and its dimensionless form is shown as (3.30).

\section{Acknowledgment}

The author is indebted to the financial support from National Science Council of Taiwan with the Grant no. NSC 97-2221-E-270-013-MY3.

\section{References}

[1] G. G. Stokes, "On the effect of the internal friction of fluids on the motion of pendulums," Transactions of the Cambridge Philosophical Society, vol. 9, pp. 8-106, 1851.

[2] R. Panton, "The transient for Stokes' oscillating plate: a solution in terms of tabulated functions," Journal of Fluid Mechanics, vol. 31, pp. 819-825, 1968.

[3] M. E. Erdogan, "Note on an unsteady flow of a viscous fluid due to an oscillating plane wall," International Journal of Non-Linear Mechanics, vol. 35, no. 1, pp. 1-6, 2000.

[4] C. M. Liu and I. C. Liu, "A note on the transient solution of Stokes' second problem with arbitrary initial phase," Journal of Mechanics, vol. 22, no. 4, pp. 349-354, 2006.

[5] C. Fetecau and C. Fetecau, "A new exact solution for the flow of a Maxwell fluid past an infinite plate," International Journal of Non-Linear Mechanics, vol. 38, no. 3, pp. 423-427, 2003.

[6] P. M. Jordan, A. Puri, and G. Boros, "On a new exact solution to Stokes' first problem for Maxwell fluids," International Journal of Non-Linear Mechanics, vol. 39, no. 8, pp. 1371-1377, 2004.

[7] M. E. Erdogan, "On unsteady motions of a second-order fluid over a plane wall," International Journal of Non-Linear Mechanics, vol. 38, no. 7, pp. 1045-1051, 2003.

[8] W. Tan and T. Masuoka, "Starting solutions for some unsteady unidirectional flows of a second grade fluid," International Journal of Engineering Science, vol. 43, no. 10, pp. 781-789, 2005.

[9] C. Fetecau and C. Fetecau, "Stokes' first problem for a second grade fluid in a porous half-space with heated boundary," International Journal of Non-Linear Mechanics, vol. 40, no. 4, pp. 515-522, 2005. 
[10] D. Vieru, T. Hayat, C. Fetecau, and C. Fetecau, “On the first problem of Stokes for Burgers' fluids. II: the cases $v=\lambda^{2} / 4$ and $v>\lambda^{2} / 4$," Applied Mathematics and Computation, vol. 197, no. 1, pp. 76-86, 2008.

[11] C. Fetecau, D. Vieru, T. Hayat, and C. Fetecau, "On the first problem of Stokes for Burgers' fluids, I: case $v<\lambda^{2} / 4, "$ Nonlinear Analysis: Real World Applications, vol. 10, no. 4, pp. 2183-2194, 2009.

[12] J. G. Oldroyd, "On the formulation of rheological equations of state," Proceedings of the Royal Society. London A, vol. 200, pp. 523-541, 1950.

[13] C. Fetecau and C. Fetecau, "The first problem of Stokes for an Oldroyd-B fluid," International Journal of Non-Linear Mechanics, vol. 38, no. 10, pp. 1539-1544, 2003.

[14] W. Tan and T. Masuoka, "Stokes' first problem for an Oldroyd-B fluid in a porous half space," Physics of Fluids, vol. 17, no. 2, Article ID 023101, 7 pages, 2005.

[15] N. Aksel, M. Scholle, and C. Fetecau, "Starting solutions for some unsteady unidirectional flows of Oldroyd-B fluids," Zeitschrift fur Angewandte Mathematik und Physik, vol. 57, no. 5, pp. 815-831, 2006.

[16] H. Qi and M. Xu, "Stokes' first problem for a viscoelastic fluid with the generalized Oldroyd-B model," Acta Mechanica Sinica, vol. 23, no. 5, pp. 463-469, 2007.

[17] K. R. Rajagopal and R. K. Bhatnagar, "Exact solutions for some simple flows of an Oldroyd-B fluid," Acta Mechanica, vol. 113, no. 1-4, pp. 233-239, 1995.

[18] T. Hayat, A. M. Siddiqui, and S. Asghar, "Some simple flows of an Oldroyd-B fluid," International Journal of Engineering Science, vol. 39, no. 2, pp. 135-147, 2001.

[19] C. M. Liu, H. H. Hwung, and C. H. Kong, "The unsteady viscous flow generated by an oscillating porous plate," Journal of Mechanics, vol. 24, no. 2, pp. 145-152, 2008.

[20] P. M. Jordan and P. Puri, "Stokes' first problem for a Rivlin-Ericksen fluid of second grade in a porous half-space," International Journal of Non-Linear Mechanics, vol. 38, no. 7, pp. 1019-1025, 2003.

[21] Y. Zeng and S. Weinbaum, "Stokes problems for moving half-planes," Journal of Fluid Mechanics, vol. 287, pp. 59-74, 1995.

[22] C. M. Liu, "Complete solutions to extended Stokes' problems," Mathematical Problems in Engineering, vol. 2008, Article ID 754262, 2008.

[23] H. A. Barnes, J. F. Hutton, and K. Walters, An Introduction to Rheology, Elsevier Science, New York, NY, USA, 1989.

[24] I. S. Gradshteyn and I. M. Ryzhik, Table of Integrals, Series, and Products, chapter, Academic Press, New York, NY, USA, 1980. 


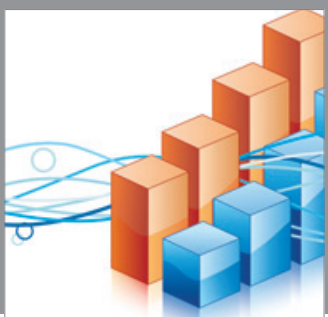

Advances in

Operations Research

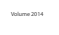

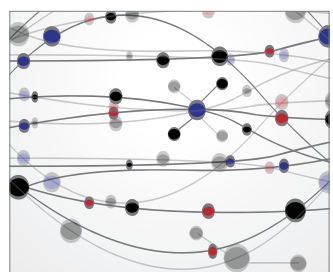

\section{The Scientific} World Journal
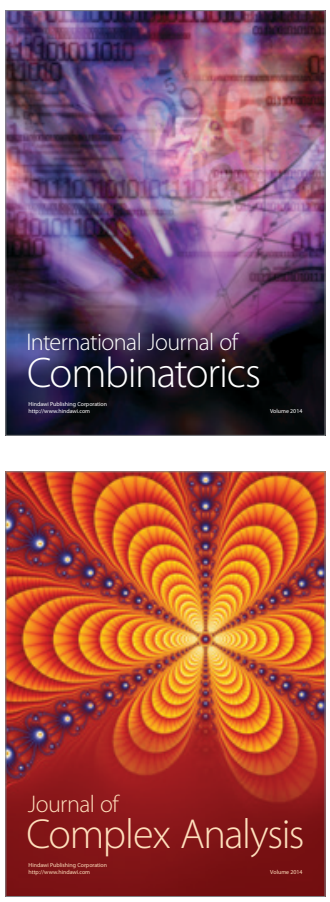

International Journal of

Mathematics and

Mathematical

Sciences
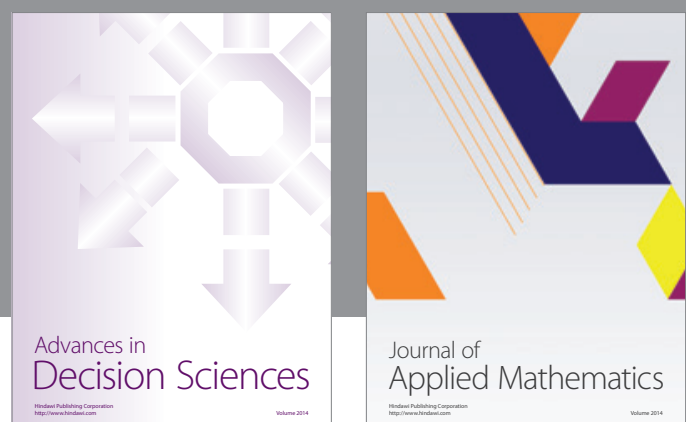

Journal of

Applied Mathematics
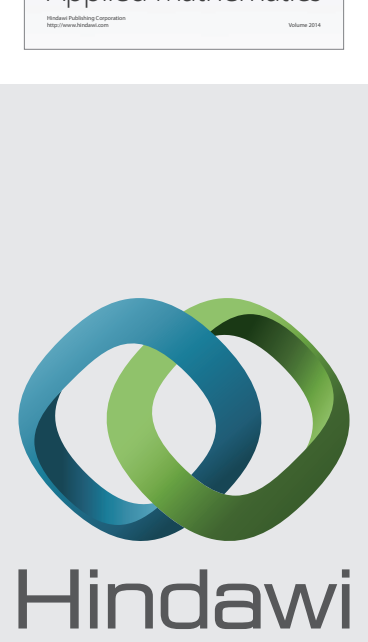

Submit your manuscripts at http://www.hindawi.com
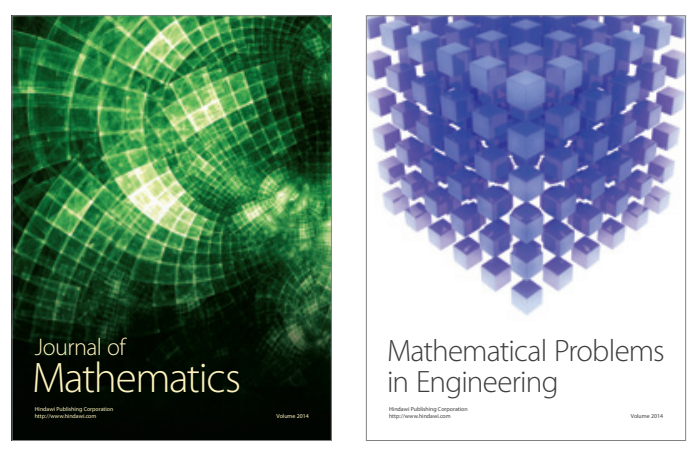

Mathematical Problems in Engineering
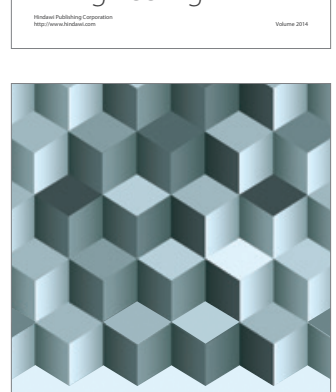

Journal of

Function Spaces
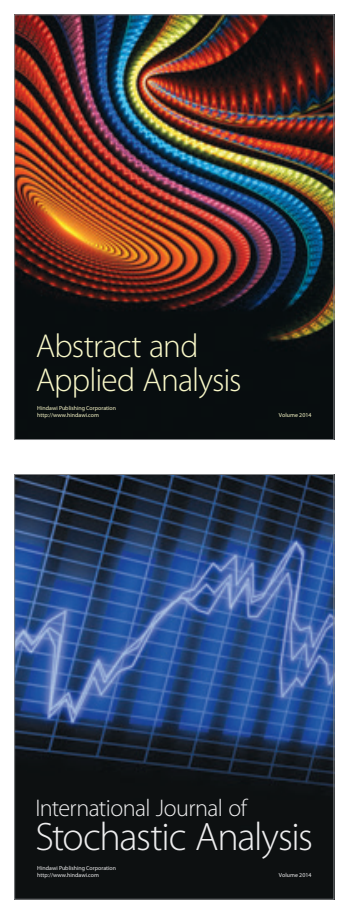

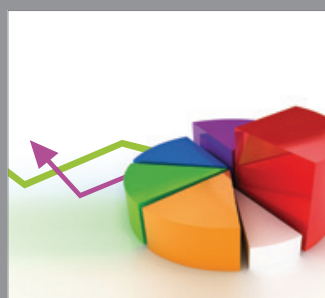

ournal of

Probability and Statistics

Promensencen
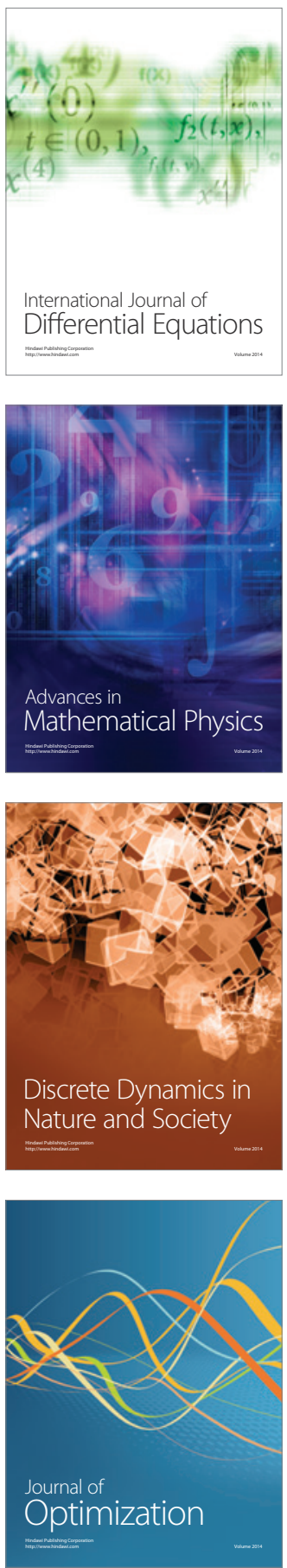